\title{
Group average representations in Euclidean distance cones
}

\author{
Casper J. Albers ${ }^{1}$, Frank Critchley ${ }^{1}$, and John C. Gower ${ }^{1,2}$ \\ 1 Department of Statistics, The Open University \\ Walton Hall, Milton Keynes MK7 6AA, United Kingdom \\ 2 Corresponding author: j.c.gower@open.ac.uk
}

\begin{abstract}
The set of Euclidean distance matrices has a well-known representation as a convex cone. The problems of representing the group averages of $K$ distance matrices are discussed, but not fully resolved, in the context of SMACOF, Generalized Orthogonal Procrustes Analysis and Individual Differences Scaling. The polar (or dual) cone representation, corresponding to inner-products around a centroid, is also discussed. Some new characterisations of distance cones in terms of circumhyperspheres are presented.
\end{abstract}

\section{Introduction}

An $n \times n$ matrix $\mathbf{D}=\left\{d_{i j}^{2}\right\}$ of squared Euclidean distances is symmetric, with zero diagonal and $m=\left(\begin{array}{c}n \\ 2\end{array}\right)$ essentially different non-negative off-diagonal values and hence may be represented by a point with coordinates $\mathbf{d}=\operatorname{vec}(\mathbf{D})$ in $m$-dimensional Euclidean space. $\mathcal{D}$ denotes the set of all such $m$-vectors d. Here, vec denotes stringing out the subdiagonal values of $\mathbf{D}$ as a vector. It is well known that $\mathbf{D}$ forms a convex cone. Writing $\mathbf{N}=\mathbf{1 1}^{\prime} / n$, the centered distance matrix $\mathbf{B}=-\frac{1}{2}(\mathbf{I}-\mathbf{N}) \mathbf{D}(\mathbf{I}-\mathbf{N})$ is symmetric with zero row-sums, and is positive semi-definite (p.s.d.) (Schoenberg (1935)). Thus, $\mathbf{B}$ is a member of $\mathcal{B}$, a sub-cone of the convex cone of all p.s.d. matrices; this result is sometimes stated as $-\mathbf{D}$ is p.s.d. on $\mathbf{x}^{\prime} \mathbf{1}=0$. Because $\mathbf{B}$ has zero row and column sums, $\mathcal{B}$ also has dimensionality $m$, a property that allows the coordinates $\mathbf{b}=\operatorname{vec}(\mathbf{B})$ to be represented, without loss of information, by only the $m$ elements below the diagonal, with a corresponding redefinition of $\mathcal{B} . \mathcal{B}$ consists of all the vectors making an obtuse angle with everything in $\mathcal{D}$, and conversely, so that the smallest angle between $\mathbf{b} \in \mathcal{B}$ and $\mathbf{d} \in \mathcal{D}$ is 90 degrees. This representation has been found useful for demonstrating some basic least-squares properties of minimising Sstress in multidimensional scaling (Critchley (1980)) and in developing MDS algorithms (Haydn et al. (1991)), whose terminology of referring to EDMs (Euclidean Distance Matrices) we adopt. Recently, Dattorro (2005) has given a masterly account of the properties of convex squared-distance cones. One line of development expresses the cone of EDMs as the intersection of two simpler convex cones, e.g. the cone of matrices p.s.d. on $\mathbf{x}^{\prime} \mathbf{1}=0$ and the cone of symmetric matrices with zero diagonal. Then, efficient algorithms such as that of Dykstra 
(1983) may be used to find the best EDM $\mathbf{D}$ that approximates any observed symmetric $\boldsymbol{\Delta}$. Similarly, Critchley (1980) noted that $\mathbf{D}$ is characterized by the properties (i) that $\delta-\mathbf{d} \in \mathcal{B}$, where $\delta=\operatorname{vec}(\boldsymbol{\Delta})$ and (ii) $\mathbf{d}^{\prime}(\delta-\mathbf{d})=0$. However, if, as is usual, one is interested in $r$-dimensional ( $r$ small $)$ approximations, we encounter difficulties because $r$-dimensional EDMs, although occurring as extremal rays of $\mathcal{D}$, do not themselves form a convex cone.

We shall not explore approximation here but shall be concerned with some problems arising from the simultaneous representation of $K$ EDMs, $\mathbf{D}_{1}, \mathbf{D}_{2}, \ldots, \mathbf{D}_{K}$. The analysis of $K$ EDMs is common in statistics where each $\mathbf{D}_{k}$ is modeled as a simple function of a common matrix $\mathbf{D}$, i.e. $\mathbf{D}_{k}=f_{k}(\mathbf{D})$ $k=1,2, \ldots, K$. The precise forms of the functions and of $\mathbf{D}$ vary among statistical methods but the central idea is that there is some group-average, represented by $\mathbf{D}$, to which each $\mathbf{D}_{k}$ is simply related. It would be interesting to know the location in the cones $\mathcal{D}$ and $\mathcal{B}$ of the group average relative to the $K$ EDMs. The methods which we examine are (i) SMACOF (Heiser and De Leeuw (1979)) (ii) Generalised Procrustes Analysis (e.g. Gower and Dijksterhuis (2005)) and Individual Differences Scaling, INDSCAL (Carroll and Chang (1972)). All these methods are well-established with supporting software. Computation of the group-averages pertaining to the different methods is not a major difficulty but our hope is that their cone representations may give further insight into the properties of such methods. Thus, this paper is concerned with the positioning of the group-average in $\mathcal{D}$ and $\mathcal{B}$; we shall see, the problem is far from trivial and much remains to be done.

Not only are EDMs, with their cones $\mathcal{D}$ and $\mathcal{B}$, important but also configurations of $n$ points that generate the EDMs. Thus, any decomposition $\mathbf{B}=\mathbf{X} \mathbf{X}^{\prime}$ gives a matrix $\mathbf{X}$ whose rows generate the distances comprising the elements of $\mathbf{D}$. Of course, $\mathbf{X}$ is determined only up to an arbitrary orthogonal transformation. How then can these configuration matrices be fitted into the cone representations? Using the singular value decomposition $\mathbf{X}=\mathbf{U} \boldsymbol{\Sigma} \mathbf{V}^{\prime}$, we note that the orthogonal transformation $\mathbf{Y}=\mathbf{X V \mathbf { U } ^ { \prime }}=\mathbf{U} \boldsymbol{\Sigma} \mathbf{U}^{\prime}$ is symmetric and, because singular values are non-negative, $\mathbf{Y}$ is p.s.d.. Furthermore, because $\mathbf{B}$ is centred, so is $\mathbf{Y}$. Thus $\mathbf{Y}$ is a member of $\mathcal{B}$. It follows that any centred configuration matrix $\mathbf{X}$ may be represented uniquely in the same cone $\mathcal{B}$ as its inner-product $\mathbf{X} \mathbf{X}^{\prime}$. Indeed because $\mathbf{X} \mathbf{X}^{\prime}=\mathbf{U} \boldsymbol{\Sigma}^{2} \mathbf{U}^{\prime}$ and $\mathbf{Y}=\mathbf{U} \boldsymbol{\Sigma} \mathbf{U}^{\prime}$ the points representing these matrices in $\mathcal{B}$ are different weighted sums of the same elementary inner-product matrices $\mathbf{u}_{r} \mathbf{u}_{r}^{\prime}$, represented by points $U_{r}$ $(r=1,2, \ldots, R)$, say, where $R$ is the dimensionality of $\mathbf{X}$. Being of deficient rank, these points are necessarily on the surface of the cone, whose interior only contains full rank matrices. The geometry is shown in Figure 1.

The linkage between the point $\mathrm{D}$ representing an EDM $\mathbf{D}$ in $\mathcal{D}$ and its inner-product counterpart $\mathbf{B}$, represented by $\mathrm{B}$ in $\mathcal{B}$, is suggested in Figure 1. The algebraic expression of the linear transformation $\mathbf{b}=\mathbf{T} \mathbf{d}$ and its inverse $\mathbf{d}=\mathbf{K t}$ have been given by Critchley (1988) and Gower and Groenen (1991) and it is not difficult to interpret them in terms of geometric orthogonal 


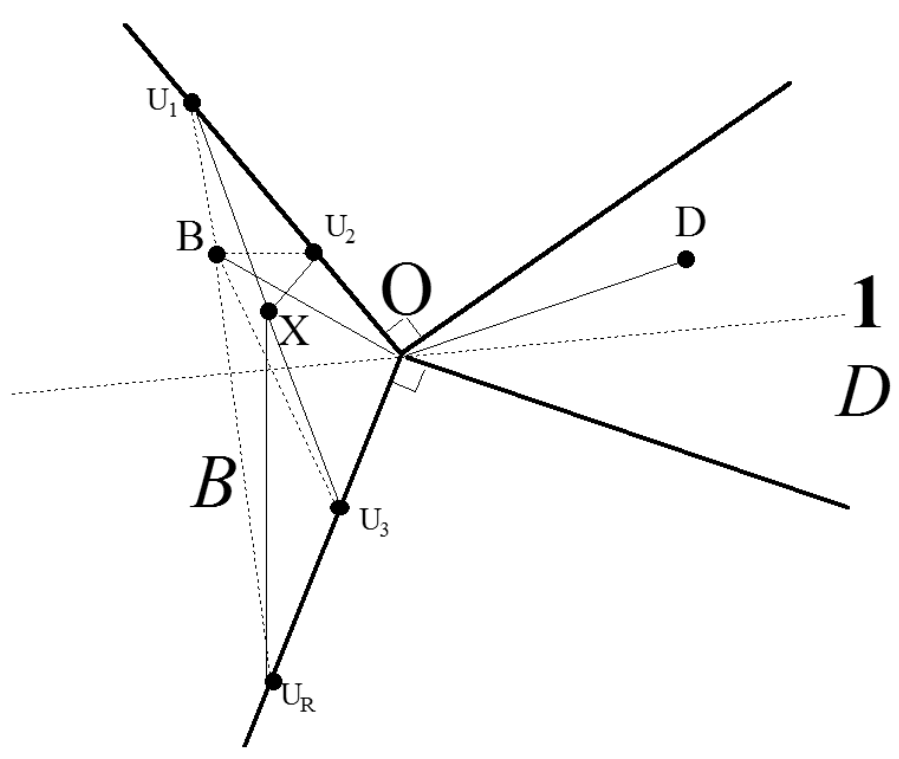

Fig. 1. The point D represents an EDM with inner-product matrix represented by the point $\mathrm{B}$. X represents the symmetricised configuration matrix that generates $\mathbf{B}$ and hence $\mathbf{D}$. B and $\mathrm{X}$ are different weighted sums of the same elementary inner-product matrices represented by $\mathrm{U}_{1}, \mathrm{U}_{2}, \ldots, \mathrm{U}_{R}$.

projections onto the direction $\mathbf{1}$, representing the unit ray of both cones, and onto an $n$-dimensional subspace orthogonal to $\mathbf{1}$ that generates the symmetric

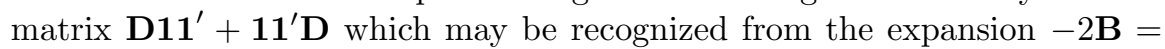
$(\mathbf{I}-\mathbf{N}) \mathbf{D}(\mathbf{I}-\mathbf{N})=\mathbf{D}-\mathbf{N D}-\mathbf{D N}+\left(\mathbf{1}^{\prime} \mathbf{D} \mathbf{1} / n\right) \mathbf{N}$. The term $\frac{1}{2}\left(\mathbf{1}^{\prime} \mathbf{D} \mathbf{1} / n\right)$ is the total sum-of-squares about their centroid of all configurations that generate $\mathbf{D}$, a quantity that is proportional to the length of the projection of $\mathbf{d}$ onto 1. Apart from this simple representation, the remainder of the geometry of projections does not seem to lend itself to elegance. Nevertheless, corresponding to every set of points in $\mathcal{B}$ is another set of points in $\mathcal{D}$ which, in principle, allows any geometry in the one space to be transformed into a dual geometry on the other space.

\section{Special cases}

We now look at some of the detailed geometry of the statistical methods under discussion.

\subsection{SMACOF}

Here, we present a variant of the method that minimises $\sum_{k=1}^{K}\left\|\mathbf{D}_{k}-\mathbf{D}\right\|^{2}$ which is, of course, given by $\mathbf{D}=\frac{1}{K} \sum_{k=1}^{K}\left(\mathbf{D}_{k}\right)$. Actually, SMACOF (Scal- 


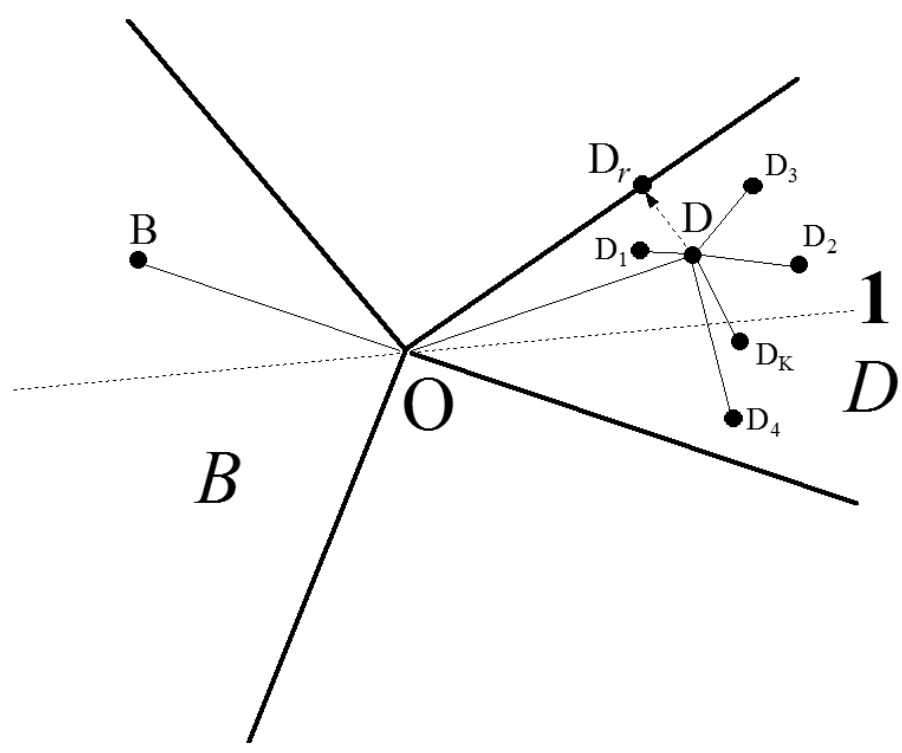

Fig. 2. SMACOF. The cone $\mathcal{D}$ of EDMs with its polar cone $\mathcal{B}$ of centred innerproduct matrices $\mathcal{B}$. The EDM $\mathbf{D}$ is shown at the centroid of $K$ EDMs. Also shown on the surface of the cone is the nearest $r$-dimensional approximation $\mathbf{D}_{r}$ to $\mathbf{D}$ together with the linkage of $\mathbf{B}$ to $\mathbf{D}$.

ing by Majorizing a Complicated Function) operates on distances and not squared distances but the mean of $K$ matrices of (unsquared) distances is not necessarily another distance matrix, implying that matrices of unsquared distances do not define a convex cone. In terms of the EDM cone $\mathcal{D}, \mathbf{D}$ is simply at the centroid of the points representing the $K$ matrices of squared distances. This is probably the simplest representation of a group-average. Of course, SMACOF, being an MDS method, is interested in $r$-dimensional configurations $\mathbf{X}$ that approximate $\mathbf{D}$ where $r$ is small. These can be found by a variety of MDS algorithms; in the case of true SMACOF by using a majorisation algorithm to minimise the Stress criterion and in our variant by minimizing Sstress. Geometrically, this means finding the nearest $r$-dimensional EDM to $\mathbf{D}$ and this lies on the surface of $\mathcal{D}$. However, corresponding to the group-averages in $\mathcal{D}$ there is a complementary set of points $\mathrm{B}_{1}, \mathrm{~B}_{2}, \ldots, \mathrm{B}_{K}$ with their group-average in $\mathcal{B}$. The geometry is shown in Figure 2.

\subsection{Generalised Procrustes Analysis (GOPA)}

Gower and Dijksterhuis (2005) discuss many variants of GPA but here we are concerned with the most popular method, Generalised Orthogonal Procrustes Analysis (GOPA) which is confined to orthogonal transformations $\mathbf{Q}_{k}(k=$ $1,2, \ldots, K)$, so preserving distances among the configurations. Specifically, we 
are given centred configurations $\mathbf{X}_{k}(k=1,2, \ldots, K)$, all assumed to have the same number of columns, and we require to find the $\mathbf{Q}_{k}(k=1,2, \ldots, K)$ that minimize:

$$
\sum_{k=1}^{K}\left\|\mathbf{X}_{k} \mathbf{Q}_{k}-\mathbf{G}\right\|^{2} \text { where } \mathbf{G}=\frac{1}{K} \sum_{k=1}^{K}\left(\mathbf{X}_{k} \mathbf{Q}_{k}\right) \text {, the group average. }
$$

As explained above, every centered configuration may be regarded as a point in $\mathcal{B}$. However, the symmetricising orientations of the configurations given by $\mathbf{V} \mathbf{U}^{\prime}$ derived from the singular value decomposition are very unlikely to coincide with those given by the optimal estimates of $\mathbf{Q}_{k}$ derived by GOPA. If the two orientations do coincide, $\mathbf{G}$ would be at the centroid as in SMACOF. We are thus led to consider where the GOPA group average lies relative to the individual configurations in the cones $\mathcal{D}$ and $\mathcal{B}$. Every configuration $\mathbf{X}_{k}$ defines a unique EDM $\mathbf{D}_{k}$, so it would be interesting to know how the EDM of the group average generated by the GOPA $\mathbf{G}$ relates to the centroid derived from SMACOF. Because the symmetricised configurations of $\mathcal{B}$ are not optimally oriented they are not likely to lead to anything useful. However, they do have a centroid which is also a member of $\mathcal{B}$. Is this configuration and the EMD it generates of any interest?

A further property of GOPA is more encouraging. It is known that necessary and sufficient conditions for the optimal GOPA fit is that $\mathbf{G}^{\prime} \mathbf{X}_{k} \mathbf{Q}_{k}$ $(k=1,2, \ldots, K)$ is symmetric and p.s.d. (see e.g. Gower and Dijksterhuis (2005)). It follows that the matrices $\mathbf{G}^{\prime} \mathbf{X}_{k} \mathbf{Q}_{k}$ are members of $\mathcal{B}$ that do incorporate their optimal GOPA rotations; these points have a centroid $\mathbf{G}^{\prime} \mathbf{G}$. All these points have their complementary EDMs in $\mathcal{D}$. Furthermore, the residual sum-of-squares arising from the $k$ th configuration is:

$$
\operatorname{trace}\left(\mathbf{X}_{k}^{\prime} \mathbf{X}_{k}+\mathbf{G}^{\prime} \mathbf{G}-2 \mathbf{G}^{\prime} \mathbf{X}_{k} \mathbf{Q}_{k}\right) \text {. }
$$

It is usual to pre-scale the data so that $\operatorname{trace}\left(\mathbf{X}_{k}^{\prime} \mathbf{X}_{k}\right)=1$, so that the first two terms are constant for all settings of $k$. The third term is the projection of $\mathbf{G}^{\prime} \mathbf{X}_{k} \mathbf{Q}_{k}$ onto the unit ray, so giving a neat geometrical representation of the residual sum-of-squares.

\section{$2.3 \quad$ INDSCAL}

This method defines a group-average configuration matrix $\mathbf{X}$ specified in a few dimensions $\mathrm{R}$ that are weighted in such a way that $\mathbf{X}$ generates an approximation to the individual centred inner product matrices $\mathbf{B}_{k}(k=$ $1,2, \ldots, K)$. Specifically, we require that approximately:

$$
\mathbf{B}_{k}=\mathbf{X W}_{k} \mathbf{X}^{\prime}
$$

where each $\mathbf{W}_{k}$ is a diagonal matrix with positive elements. Usually, the approximations are found by minimizing $\sum_{k=1}^{K}\left\|\mathbf{B}_{k}-\mathbf{X W}_{k} \mathbf{X}^{\prime}\right\|^{2}$ using an 


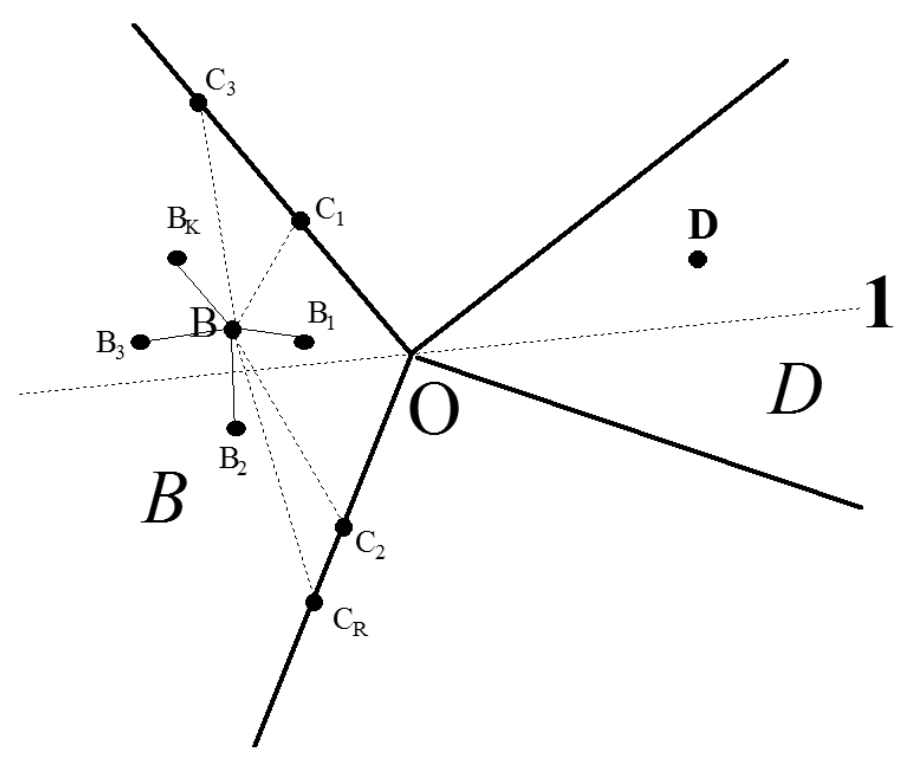

Fig. 3. The inner-product matrices $\mathbf{B}_{1}, \mathbf{B}_{2}, \ldots, \mathbf{B}_{K}$ are different weighted means of the elementary group-average inner-product matrices $\mathbf{C}_{1}, \mathbf{C}_{2}, \ldots, \mathbf{C}_{R}$; the groupaverage $\mathbf{B}$ is itself an unweighted mean of these points. A similar geometry exists in $\mathcal{D}$ but for simplicity is not shown in detail.

ALS algorithm called CANDECOMP (Carroll and Chang, 1972). So far as the cone $\mathcal{B}$ is concerned, each column of $\mathbf{X}$ defines an elementary inner-product matrix $\mathbf{C}_{r}=\mathbf{x}_{r} \mathbf{x}_{r}^{\prime}$ represented by a point $C_{r}(r=1,2, \ldots, \mathrm{R})$. Thus, each $\mathbf{B}_{k}$ is at a weighted mean $\sum_{r=1}^{\mathrm{R}} w_{k r} \mathbf{C}_{r}$ while unit weights generate a groupaverage inner product matrix $\mathbf{B}=\mathbf{X X}^{\prime}$. Figure 3 illustrates the geometry, where dotted lines indicate the group-average $\mathbf{B}$. Similar lines may be thought of as joining each $\mathbf{B}_{k}$ to $\mathrm{C}_{1}, \mathrm{C}_{2}, \ldots, \mathrm{C}_{K}$.

\section{Other Representations}

In the above, we have concentrated on the relationship between $\mathcal{D}$ and $\mathcal{B}$ defined by $\mathbf{B}=-\frac{1}{2}(\mathbf{I}-\mathbf{N}) \mathbf{D}(\mathbf{I}-\mathbf{N})$ or, equivalently, by $\mathbf{B}=-\frac{1}{2}\left(\mathbf{I}-\mathbf{1} \mathbf{s}^{\prime}\right) \mathbf{D}(\mathbf{I}-$ $\left.\mathbf{s} \mathbf{1}^{\prime}\right)$ where $\mathbf{s}=\mathbf{1} / n$. It is this choice of $\mathbf{s}$ that ensures that the row and columns sums of $\mathbf{B}$ are zero and that generating coordinates of $\mathbf{X}$ are centered at the centroid. This choice also defines a linear transformation of $\mathbf{D}$ and ensures that the cones $\mathcal{D}$ and $\mathcal{B}$ are orthogonal. Despite these nice properties it may be worth considering other choices of $\mathbf{s}$ that give different centrings (Gower (1982)). Of special interest is to choose $\mathbf{s}$ to centre at the circumcentre. Gower (1985) showed that: for every EDM $\mathbf{D}$, there exists a circumhypersphere iff $\mathbf{1}^{\prime} \mathbf{D}^{-} \mathbf{1} \neq 0$ given by $\mathbf{s}=\mathbf{D}^{-} \mathbf{1} /\left(\mathbf{1}^{\prime} \mathbf{D}^{-} \mathbf{1}\right)$. This has radius $\mathrm{R}^{2}=-\left(\mathbf{1}^{\prime} \mathbf{D}^{-} \mathbf{1}\right)^{-1}$. 
Furthermore, if $\mathbf{1}^{\prime} \mathbf{D}^{-} \mathbf{1}=0$ there exists a $g$-circumhypersphere 0 given by $\mathbf{s}=\left(\mathbf{D}^{2}\right)^{-} \mathbf{1} /\left(\mathbf{1}^{\prime}\left(\mathbf{D}^{2}\right)^{-} \mathbf{1}\right)$. This has radius $\mathbf{R}^{2}=\left(\mathbf{1}^{\prime}\left(\mathbf{D}^{3}\right)^{-} \mathbf{1}\right) /\left(\mathbf{1}^{\prime}\left(\mathbf{D}^{2}\right)^{-} \mathbf{1}\right)^{2}$.

Here, $\mathbf{D}^{-}$represents any $g$-inverse of $\mathbf{D}$, and a $g$-circumhypersphere is one whose radius minimizes the sum-of-squares of the differences between a sphere and the actual, unequal, squared radii. Thus, we have three situations, (i) D is non-singular, has a circumhypersphere, and, as usual lies in the interior of the cone $\mathcal{D}$, (ii) $\mathbf{D}$ is singular so lies on the exterior of $\mathcal{D}$ but $\mathbf{1}^{\prime} \mathbf{D}^{-} \mathbf{1} \neq 0$, and so there continues to be a true circumhypersphere and (iii) $\mathbf{1}^{\prime} \mathbf{D}^{-} \mathbf{1}=0$ so $\mathbf{D}$ lies on the exterior of $\mathcal{D}$ and there is no circumhypersphere, although there is a $g$-circumhypersphere. One may say that when $\mathbf{1}^{\prime} \mathbf{D}^{-} \mathbf{1}=0$, the circumhypersphere has infinite radius, so that the generating coordinates lie on a flat. An important property is that circumcentres are defined for all points in the interior of $\mathcal{D}$ and for some points on extremal rays.

Substituting for $\mathbf{s}$ the transformation is found to have the particularly simple form $\mathbf{C}=-\frac{1}{2} \mathbf{D}+\mathrm{R}^{2} \mathbf{1} \mathbf{1}^{\prime}$ but this does not represent a linear function because $\mathrm{R}$ is the above-mentioned function of $\mathbf{D}$. The dimensionality of $\mathbf{C}$ remains $m$ and, as with $\mathbf{B}$, we may work with $\mathbf{c}=\operatorname{vec}(\mathbf{C})$. Next, we show how to recover the complete form of $\mathbf{D}$, at least for interior points of $\mathcal{D}$, from the sub-diagonal elements, $\mathbf{C}_{0}$, of $\mathbf{C}$. We know that $\mathbf{C}=-\frac{1}{2}\left(\mathbf{I}-\mathbf{1} \mathbf{s}^{\prime}\right) \mathbf{D}\left(\mathbf{I}-\mathbf{s 1}^{\prime}\right)$ for some $\mathbf{s}=\mathbf{D}^{-} \mathbf{1} /\left(\mathbf{1}^{\prime} \mathbf{D}^{-} \mathbf{1}\right)$ and some unknown $\mathbf{D}$, which we require to construct. Thus, $\mathbf{C}=\mathbf{C}_{0}+\mathrm{K} \mathbf{I}$ for some $\mathrm{K}$ equal to the unknown $\mathrm{R}^{2}$. Because $\mathbf{C s}=0$, it follows that $\mathbf{C}_{0} \mathbf{s}=-K \mathbf{s}$ and we may set $\mathbf{K}=-\lambda$ where $\lambda$ is the smallest (necessarily negative) eigenvalue of $\mathbf{C}_{0}$. Note that this setting ensures that $\mathbf{C}$ is p.s.d., which it would not be if any other negative eigenvalue were chosen. Having identified $\mathrm{R}^{2}$ we may construct $\mathbf{C}=\mathbf{C}_{0}+\mathrm{R}^{2} \mathbf{I}$ and $\mathbf{D}=2\left(\mathrm{R}^{2} \mathbf{1 1} \mathbf{1}^{\prime}-\mathbf{C}\right)=2\left\{\mathrm{R}^{2}\left(\mathbf{1 1}^{\prime}-\mathbf{I}\right)-\mathbf{C}_{0}\right\}$.

The above shows that every $\mathbf{C}_{0}$ corresponds to a unique EDM $\mathbf{D}$. To increase understanding of this geometry, we investigate contours of constant $\mathrm{R}^{2}$ in $\mathcal{D}$. A full study is not possible here and we content ourselves with examining the cross-section of $\mathcal{D}$ that contains the unit ray $\mathbf{1}$ and the fundamental rank-2 EDM which consists of two sets of $p$ and $q(n=p+q)$ points coincident at points $\mathrm{P}$ and $\mathrm{Q}$, say, respectively. We assume that $\mathrm{P}$ and $\mathrm{Q}$ are unit distance apart, defining a vector $\mathbf{r}$ giving the squared distances (all equal to unity or zero). Although $\mathbf{D}$ is only of rank-2, $\mathrm{P}$ and $\mathrm{Q}$ lie on a 'circle' centred at their midpoint and so have a circumcircle with $\mathrm{R}^{2}=\frac{1}{4}$. Other points $\lambda \mathbf{r}$ on the same ray will replace $R^{2}$ by $\lambda R^{2}$. The unit ray 1 corresponds to an EDM of a regular simplex and has $\mathbf{R}^{2}=(n-1) / 2 n$. Also, $\mathbf{r}$ has $p q$ unit values so that $\mathbf{r}^{\prime}(\mathbf{r}-\mathbf{1})=0$, showing that $\mathbf{r}$ is the orthogonal projection of $\mathbf{1}$ onto the ray through $\mathbf{r}$. This is shown in Figure 4(a), which gives the basic geometry of the cross-section of the EDM cone. We wish to find the circumradius of any EDM in the plane defined by $\mathbf{1}$ and $\mathbf{r}$. Writing $\mathbf{D}_{1}$ and $\mathbf{D}_{2}$ for the matrix 
forms of these EDMs, we have:

$$
\begin{aligned}
-2 \mathbf{D} & =-2\left(\lambda \mathbf{D}_{1}+\mu \mathbf{D}_{2}\right)=\lambda\left(\mathbf{I}-\mathbf{1 1}^{\prime}\right)-\mu\left(\begin{array}{cc}
\mathbf{0} & \mathbf{1 1}^{\prime} \\
\mathbf{1 1 ^ { \prime }} & \mathbf{0}
\end{array}\right) \\
& =\left(\begin{array}{cc}
\lambda\left(\mathbf{I}-\mathbf{1 1 ^ { \prime }}\right) & -(\lambda+\mu) \mathbf{1 1 ^ { \prime }} \\
-(\lambda+\mu) \mathbf{1 1 ^ { \prime }} & \lambda\left(\mathbf{I}-\mathbf{1 1}^{\prime}\right)
\end{array}\right)
\end{aligned}
$$

where the lengths of the vectors $\mathbf{1}$ are assumed defined by context and where, without loss of generality, we have assumed that the $p$ and $q$ points are labeled consecutively. After detailed algebraic manipulations we find that the circumradius $R_{\lambda \mu}^{2}$ of $\left(\lambda \mathbf{D}_{1}+\mu \mathbf{D}_{2}\right)$ is given by:

$$
2 R_{\lambda \mu}^{2}=\lambda+\frac{\mu^{2} p q-\lambda^{2}}{2 \mu p q+n \lambda}=\mu\left(\frac{\lambda}{\mu}+\frac{p q-\lambda^{2} / \mu^{2}}{2 p q+n \lambda / \mu}\right) .
$$

The right-hand form is valid only when $\mu \neq 0$, when it shows that for constant $\lambda / \mu$ the value of $\mathrm{R}^{2}$ increases proportionally to $\mu$. This result merely confirms that $\mathrm{R}^{2}$ increases with $\mu$ as one proceeds along the ray defined by the ratio $\lambda / \mu$. When $\lambda=0$ and $\mu=1$ we define $\mathbf{r}$ and correctly obtain $\mathrm{R}^{2}=\frac{1}{4}$; when $\lambda=1$ and $\mu=0$ we define $\mathbf{1}$ and correctly obtain $\mathrm{R}^{2}=n(n-1) / 2$. When $2 p q+n \lambda / \mu=0$ the circumradius becomes infinite, so $\lambda / \mu=-2 p q / n$ defines the extremal ray other than $\mathbf{r}$ the cross-section under consideration, as is shown in Figure 4(a).

We can derive the contours for constant $\mathrm{R}^{2}=\frac{1}{4}$, other contours are easily obtained by proportion. The values of $p$ and $q$ affect these contours. Generally the contour is hyperbolic as is shown in Figure 4(d) for $p=2$ and $q=6$. One branch of the hyperbola is outside the cone so is irrelevant. For emphasis we have high-lighted the part of the relevant branch that is inside the cone. This state of affairs is modified when $p=1$ or $p=q$. When $p=1$ the formula simplifies to:

$$
2 R_{\lambda \mu}^{2}=\frac{(\lambda+\mu)^{2}(n-1)}{n \lambda+2(n-1) \mu}
$$

which represents a parabola, shown in Figure 4(b) for $p=1$ and $q=7$. When $n$ is even and $p=q, \mu p+\lambda$ is a common factor and provide this is not zero we have:

$$
2 R_{\lambda \mu}^{2}=\frac{(2 p-1) \lambda+\mu p}{2 p}
$$

which represents a single straight line; the contours are then a set of parallel lines which may be shown to be orthogonal to the unit ray; the extremal ray $\mu p+\lambda, R_{\infty}^{2}$, provides a further, pathological, contour. This is shown in Figure 4(c) for $p=q=4$.

Although Figure 4 is based on the case $n=8$, the results are completely general. Of course, different labeling of the $n$ points will give different rays but their geometry is identical. Also, different settings of $p$ and $q$ will change scales but not the more fundamental geometry of hyperbolas, parabolas and pairs of 

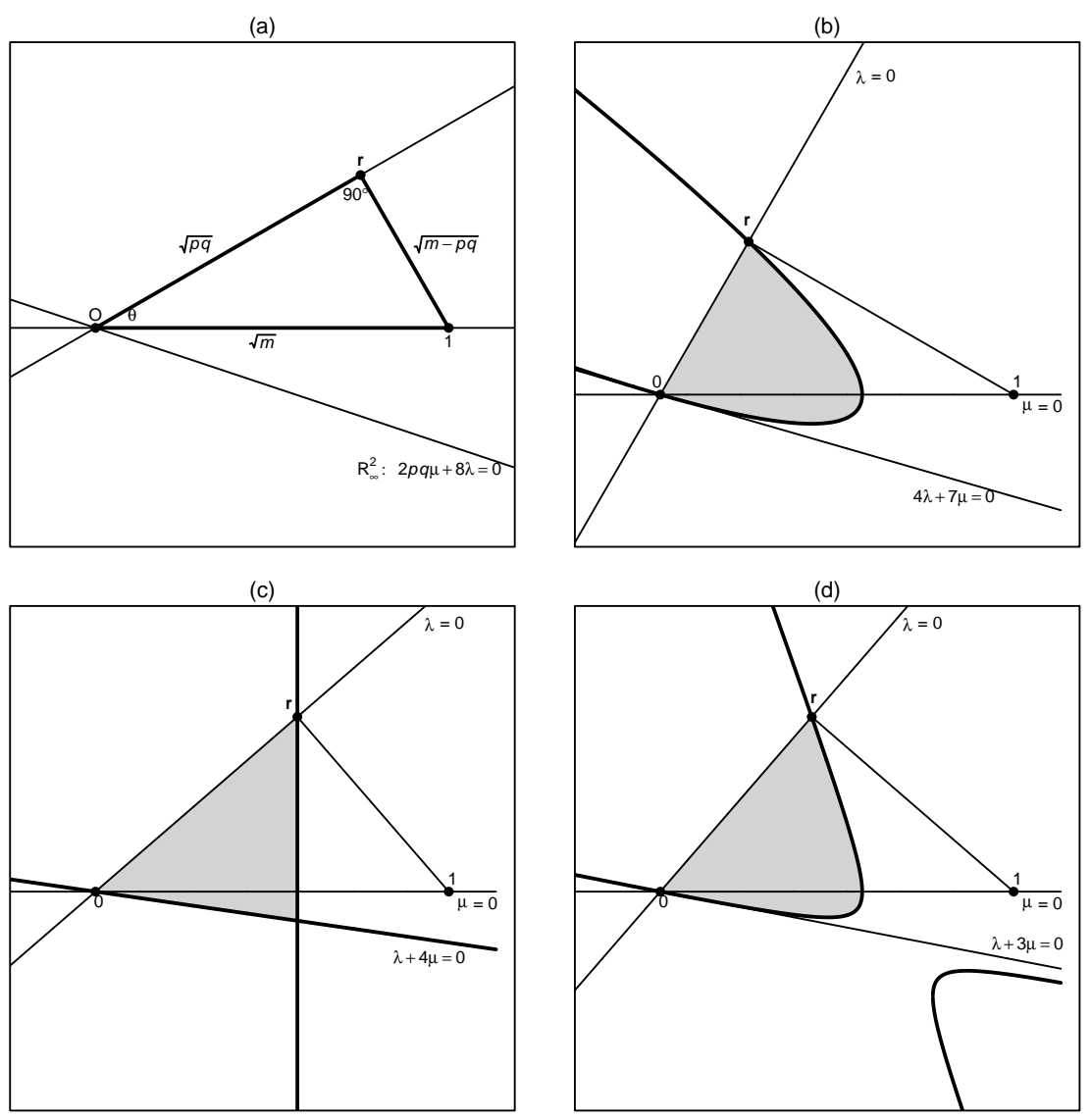

Fig. 4. Figure (a) shows the basic geometry of the cross-section of the EDM cone containing the vectors $\mathbf{r}$ and $\mathbf{1}$. The remaining figures give contours of constant circumradius $\mathrm{R}^{2}=\frac{1}{4}$ for various choices of $p$ and $q$ where $p+q=n=8$. We introduce $m=n(n+1) / 2=28$. The part of the contour in the EDM cone is highlighted in grey. Figure (d) shows the usual hyperbolic contour found (here $p=2$ and $q=6$ ). Figures (b) and (c) show the special parabolic and linear solutions found when $p=1$ and $p=q$, respectively.

lines. These results confirm the complexity of the geometry of the EDM cone, especially in the vicinity of the extremal rays. The formula $\mathbf{C}=-\frac{1}{2} \mathbf{D}+\mathrm{R}^{2} \mathbf{1 1} \mathbf{1}^{\prime}$ readily allows the contours in $\mathcal{D}$ to be transformed into contours in a space $\mathcal{C}$, analogous to the cone $\mathcal{B}$. One merely has a reflection of an $\operatorname{EDM} \frac{1}{2} \mathcal{D}$ in the origin, together with a translation $\mathrm{R}^{2}$ in the direction of the unit ray. Unfortunately, $\mathrm{R}^{2}$ varies with $\mathbf{D}$ but it is constant along the contour for constant $\mathrm{R}^{2}$, so scaled versions of the same contours persist. It seems that although $\mathcal{C}$, like $\mathcal{B}$ is part of the cone of p.s.d. matrices, it is not itself a cone. 
However, in the context of group average representations things are similar to the representations in $\mathcal{B}$. The main change is that configuration matrices $\mathbf{X}_{k}$ first have to be centred at their circumcentre before being symmetricised by rotating their singular value forms. There is then a special difficulty when a circumcentre does not exist when the translation term becomes infinite. This situation will be common when $\mathbf{X}_{k}$ is a data-matrix but not when $\mathbf{X}_{k}$ is derived from similarities. The spaces $\mathcal{C}$ and $\mathcal{D}$ are not orthogonal and, indeed, may intersect.

\section{References}

CARROLL, J. D. and CHANG, J. J. (1972): Analysis of individual differences in multidimensional scaling via an $n$-way generalization of Eckart-Young decomposition. Psychometrika 35, 283-319.

CRITCHLEY, F. (1980): Optimal norm characterisations of multidimensional scaling methods and some related data analysis problems. In: E. Diday et al. (Eds.): Data Analysis and Informatics. North Holland, Amsterdam, 209-229.

CRITCHLEY, F. (1988): On certain linear mappings between inner-products and squared-distance matrices. Linear Algebra and its Applications 105, 91-10\%.

DATTORRO, J. (2006): Convex optimization and Euclidean distance geometry. Meboo Publishing, Palo Alto, California, USA.

DYKSTRA, R. L. (1983): An algorithm for restricted least squares regression. Jounrnal of the American Statistical Association 78, 837-842.

GOWER, J.C. (1982): Euclidean distance geometry. The Mathematical Scientist 7, $1-14$.

GOWER, J.C. (1985): Properties of Euclidean and non-Euclidean distance matrices. Linear Algebra and its Applications 67, 81-97.

GOWER, J. C. and DIJKSTERHUIS, G. B. (2005): Procrustes Problems. Oxford University Press, Oxford, UK.

GOWER, J. C. and GROENEN, P. J. F. (1991): Applications of the modified Leverrier-Faddeev algorithm for the construction of explicit matrix spectral decompositions and inverses. Utilitas Mathematica 40, 51-64.

HEISER, W. and DE LEEUW, J. (1979): How to use SMACOF-1, A program for metric muktidimensional scaling. Department of Datatheory, Faculty of Social Sciences, University of Leiden, Wassenaarseweg 80, Leiden, The Netherlands, $1-63$.

HAYDN, T. L., WELLS, J. LIU, W-M and TARAZAGA, P. (1991): The cone of distance matrices. Linear Algebra and its Applications 144, 153-169.

SCHOENBERG, I. J. (1935) Remarks to Maurice Frechet's article "Sur la definition axiomatique d'une classe d'espaces vectoriels distancies applicables vectoriellement sur l'espace de Hilbert". Annals of Mathematics 36, 724-732. 